\title{
La place de La comédie des quatre femmes de Marguerite de Navarre (1542) dans le discours sur le célibat volontaire comme modèle de félicité de l'Arioste à Gabrielle Suchon ${ }^{1}$
}

BRENDA

DUNN-LARDEAU

Summary: In the Comedy for Four Women (1542), Marguerite de Navarre presents a dialogue among five female protagonists who express the delights and the torments that stem from the love of their suitors or husbands. This is true for all except the First Girl, who does not want to have anything to do with love or marriage. This article examines the special status of the character of the First Girl, an apparent forerunner of a new way of life for women. Specifically, it explores the potential for a sixteenth-century young woman to live a life without a suitor and, moreover, to proclaim that wilful celibacy is a source of genuine happiness rather than of the humiliating feeling of failure attached traditionally to a life outside of wedlock or religion. Moreover, in this play, Marguerite de Navarre makes a significant contribution to the idea of the dignity and happiness of wilful celibacy, while taking part in the Querelle des amies debate. Finally, this article gathers data on the historical and fictional characters that emerged before and after Marguerite's Comedy, leading to the development of a positive view of the unmarried woman, which culminated in Gabrielle Suchon's manifesto, Du célibat volontaire, in 1700. The data reflect a growing demographic trend, as well as a shift in the hierarchy of female values: chastity is no longer an end in itself, the supreme feminine virtue, but a means to the end of freedom and felicity.

ans la Comédie des quatre femmes de M. de Navarre, pièce pleine de Derve et d'entrain, de 1542, il y a d'abord deux jeunes filles aussi exaltées l'une que l'autre. La première fait l'éloge de la liberté et la seconde, celui de l'amour ${ }^{2}$. Ensuite, deux femmes mariées décrivent les ennuis de leur vie conjugale. Face à ces quatre personnages, une vieille expérimentée 
accepte d'écouter leur cas à la manière d'un débat médiéval, où un juge arbitre entre les parties. Cependant, contrairement à la tradition médiévale du débat, filles comme femmes refusent d'écouter les vérités que la Vieille leur dévoile et l'avenir qu'elle leur prédit. La comédie s'achève néanmoins joyeusement avec la Vieille entraînée dans le mouvement de la danse par un vieil homme et des jeunes gens dans cette sorte de momerie de carnaval.

Il est frappant que tous les personnages féminins de cette comédie s'épanchent tantôt sur les délices, tantôt sur les tourments passés, présents ou à venir que leur procure l'amour de leur ami ou de leur mari, sauf le personnage de la Première fille, comme la nomme sans plus de précision Marguerite, qui ne veut rien savoir d'Éros.

Cette étude se propose d'analyser le statut spécial de la Première fille dans ce débat. Ce personnage apparaît comme le précurseur d'un nouveau modèle de vie féminin, celui où une jeune fille refuse l'amour et le mariage, pour clamer, de surcroît, que le célibat par choix lui procure un grand bonheur et non le sentiment humiliant d'échec personnel ou la désapprobation sociale attendus.

Comment, dès lors, s'élabore ce modèle de félicité féminine honorable, qui ne passe pas par Éros, comme pour les autres personnages de cette pièce ? Chemin faisant, il y aura lieu d'apprécier la contribution de ce personnage à la conception du bonheur féminin jusque là ainsi qu'à la Querelle des amies, déclenchée par L'Amye de court (1542) de Bertrand de la Borderie. Cet article sera aussi l'occasion d'amorcer une synthèse des jalons les plus importants d'une conception positive du célibat volontaire, avant et après cette comédie de Marguerite, qui se fraye un chemin, petit à petit, dans les realia ainsi que la fiction jusqu' au manifeste de Gabrielle Suchon Du célibat volontaire en 1700 .

\section{Perspectives de bonheur pour la jeune fille au Moyen Âge et à la Renaissance}

Comte tenu, d'une part, du thème philosophique antique des genres de vie et, de l'autre, des trois âges de la femme, quelles possibilités de bonheur s'offrent aux jeunes filles du Moyen Âge et de la Renaissance ? Si la philosophie antique réussissait à réduire la vie humaine le plus souvent à trois modèles de vies : contemplative, active et voluptueuse ${ }^{3}$, Fulvia de Luise et Giuseppe Farinetti font un constat désenchanté de la place des femmes dans ces schémas dans leur Storia della felicità. Gli antichi et $i$ moderni :

Le bonheur philosophique [antique] est, dans la presque totalité de ses variantes, une proposition exclusivement au masculin. L'homme idéal n'est jamais une 
femme et tout ce qu'on lui reconnaît comme sujet actif et conscient, protagoniste de sa vie et titulaire de ses désirs, renvoie dans l'ombre le féminin, à l'arrière-plan la passive, patiente, accueillante présence des femmes. Leur silence pèse sur l'imaginaire de la félicité. [...] ce sont des hommes qui parlent à des hommes sans jamais sortir d'eux-mêmes. Une autre histoire du bonheur pourrait être écrite ${ }^{4}$.

Une exception notable à ce silence sur le bonheur féminin est la contribution au $\mathrm{XV}^{\mathrm{e}}$ siècle de Christine de Pizan ${ }^{5}$. Son Livre des trois vertus propose, certes, une définition du bonheur par la négative, en cherchant une règle de vie qui évite à toutes « un châtiment dans l'au-delà », mais qui n'occulte pas la variété des situations ${ }^{6}$.

Puis, avec la littérature morale et politique du XVIe siècle, s'élaborent «d'autres modèles de vie, de nouveaux "types" moraux et sociaux », le plus prestigieux étant celui du Livre du courtisan (1528) de Castiglione7. Quant au modèle de la courtisane dans ce manuel de savoir-vivre, il va catalyser la Querelle des amies, les uns critiquant son ficinisme idéalisé, les autres le louant.

\section{Le souverain bien pour la Première fille}

Par opposition au contexte renaissant, riche d'éthiques, le souverain bien pour la Première fille de la Comédie n'est ni le plaisir épicurien ni la vertu stoïcienne, encore moins le souci du bonheur collectif aristotélicien, non plus un ardent désir de la vision béatifique chrétienne post-mortem. Son discours est émaillé, toutefois, d'emprunts à des valeurs stoïciennes, l'austérité en moins.

D'entrée de jeu, la Première fille revendique un modèle de vie nouveau et dérangeant, c'est-à-dire celui du célibat féminin librement consenti :

Quant est de moy, j'ai mise mon estude

D'avoir le corps, et le cœur libre et franc. (v. 7-8)

Pour cette raison, elle ne place pas Plaisir et Passion en l'amour comme la Seconde fille de la Comédie, mais « tout plaisir »dans la liberté, en insistant, dès l'incipit, sur l'argument de nature :

Tout le plaisir, et le contentement, Que peult avoir un gentil cœur honneste, C'est liberté de corps, d'entendement, Qui rend heureux tout homme, oyseau, ou beste. (v. 1-4)

Si, pour le stoïcien, la liberté consiste à se conformer à l'ordre universel raisonnable et que cette acceptation du destin mène au souverain bien, la 
tranquillité de l'âme, il faut admettre que cette fille a retenu quelque chose de leur discours.

La Première fille recherche un bien inaliénable, c'est-à-dire qui ne dépende pas de la volonté d'un autre, mais de la sienne propre pour garder sa tranquillité d'âme ou « repos » comme elle le nomme, écartant tout ce qui l'en détourne :

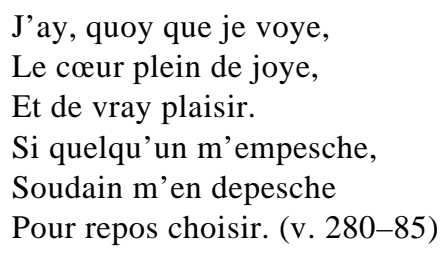

Et pour arriver à ce « repos », elle refuse d'entrer dans le jeu de la contrainte de la réciprocité courtoise :

J'ayme mon repos :

Je fuy les propos

D'amour, et sa bande.

Et qui me prieroit

D'aymer, il n'auroit

Rien que sa demande. (v. 286-91)

Précisons que ce refus d'amour se distingue de celui des Cent ballades d'amant et de dame (1394-1410) de Christine de Pizan, ainsi que de celui de La belle dame sans mercy (1424) d'Alain Chartier (pour citer un exemple canonique), car il ne s'agit plus simplement de critiquer la contrainte de la réciprocité de l'idéologie amoureuse courtoise, mais de passer outre, porté par le désir de réalisation personnelle.

Qu'à cela ne tienne, la Première fille se défend de tomber dans le défaut de «philautie » (amour de soi), tant décrié par les humanistes, ou d'indifférence aux ennuis des autres. Un brin élitiste, cependant, sa compassion va dépendre du mérite d'autrui :

J'ay bien grand desir,

De faire plaisir,

A qui le merite.

Desolation

Par compassion

A joye je incite. (v. 316-21) 


\section{La jeune fille et le célibat comme genre de vie}

Mais comment une jeune fille de la Renaissance peut-elle soutenir que le souverain bien est la liberté et prétendre refuser l'amour, quand l'histoire sociale et littéraire nous apprend que jusqu'au début de la Renaissance, les femmes ne pouvaient se réaliser que dans le mariage ou la vie conventuelle ${ }^{8}$, sauf exceptions. Margaret King est on ne peut plus claire sur l'espace social peu confortable qui attendait celles qui s'aventuraient hors des sentiers battus : «La vie sociale ne comprenait pas de catégorie pour la femme non mariée en dehors de la vie religieuse ; les femmes qui choisissaient cette voie se retrouvaient dans de pénibles limbes ${ }^{9}$. En l'absence d'une histoire du célibat féminin volontaire (qui n'est pas synonyme de celle de la chasteté), il demeure difficile de cerner avec exactitude les chances réelles du célibat laïque à l'époque de Marguerite de Navarre, car la critique s'intéresse depuis peu à différents aspects de cette problématique en diachronie comme en synchronie ${ }^{10}$.

Au surplus, parmi les exceptions dans la vie privée et publique, il faut distinguer des cas très différents. Que l'on pense à Jeanne d'Arc ou à Élisabeth Ire, la chasteté semble être le gage de leur autorité morale et politique ${ }^{11}$. Si l'on considère, par contre, les rares cas isolés rencontrés dans la vie privée et valorisés socialement, pour certains, d'autres finalités que le pouvoir se dessinent. Par exemple, Isotta Nogarola (1418-66), élevée dans un esprit humaniste, savait le grec et le latin. Celle-ci brilla dans sa jeunesse par ses traductions et poésies et resta célibataire par choix. Encore qu'il faille préciser, que le choix de se retirer dans la maison familiale, dans un «book-lined cell », selon la formule bien trouvée de Margaret King, pour y étudier les lettres sacrées plutôt que profanes de sa jeunesse, aurait aussi été motivé par le souci de faire taire les attaques sur sa réputation ${ }^{12}$.

Toutefois, ces exceptions ne devraient pas faire écran au fait qu'il semble bien exister un écart entre nos perceptions entourant l'état de quelques célibataires jouissant de notoriété et la réalité statistique de ce phénomène, surtout urbain et plus marqué chez les pauvres. Ce décalage s'expliquerait en partie, selon l'étude de synthèse de Scarlett BeauvaletBoutouyrie, en raison de l'accès difficile à des sources sur ces femmes « souvent marginales, souvent pauvres, souvent sans personne qui ait préservé leur mémoire, les femmes seules, surtout célibataires, échappent en partie au regard de l'Histoire » et également du fait de la valorisation de la mère qui fait ignorer les autres cas de figure ${ }^{13}$. En effet, les recherches suggèrent que les célibataires ne sont pas des exceptions sur le plan démographique, si l'on veut bien considérer leur place dans la domesticité d'alors, par exemple, de telle sorte que jusqu'au XVIII siècle, «selon les pays, le 
célibat définitif touche entre $10 \%$ et $20 \%$ de la population féminine. C'est à partir du milieu du XVe siècle que la proportion de femmes célibataires progresse dans toute l'Europe du Nord »14. Il reste que, de façon générale, la femme célibataire demeure suspecte, car elle menace l'ordre social et risque de mener une vie immorale ${ }^{15}$.

La littérature, pour sa part, n'a accordé que peu d'attention à la jeune fille. Sans prétendre à l'exhaustivité, voici quelques jalons de la lente émergence de ce personnage de la jeune fille qui exprime peu à peu ses aspirations. Quasi absente de l'épopée, songeons à La chanson de Roland où le héros éponyme meurt en regrettant jusqu'à son épée sans une pensée pour sa fiancée Aude, la jeune fille devient plus présente dans le roman médiéval, même si celle-ci y fait surtout figure de pièce maîtresse du jeu des alliances matrimoniales ${ }^{16}$. Et quand bien même des aspirations individuelles à l'indépendance se manifestent, c'est pour les voir niées au profit de motifs supérieurs du bien collectif ou bien soumises à l'autorité implacable des Fata. C'est le cas dans la Teseide (1340-42) de Boccace où les héroïnes mythologiques ont fait le vœu de servir la déesse Diane et de vivre sans hommes. Toutefois, dès la fin du Chant 1, Hyppolyté épousera le Grec Thésée pour éviter une guerre sans merci contre les Amazones sur lesquelles elle règne. Au Chant 7, sa jeune sœur Émilia entendra, suite à ses prières à l'autel de Diane, la prédiction voulant qu'elle épouse Palémon ou Arcita qui se battent pour son amour. Émilia aura beau résister à cette destinée jusqu'au Chant douzième et final en réitérant sa volonté de continuer à servir Diane et de mourir dans son temple ; il lui faudra se marier. Symboliquement, ce mythe, qui sera repris, entre autres, par Chaucer dans son Knight's Tale, décrit au-delà de la lutte entre Mars et Venus, la vaine volonté d'indépendance de ces héroïnes obligées, bon gré, mal gré, de servir Junon plutôt que Diane, c'est-à-dire de se conformer à l'ordre social. Au XVe siècle, les pucelles du Livre des trois vertus se préparent à leurs futures responsabilités d'épouses et de mères ; et, quoique Christine de Pizan n'y occulte pas le sort des femmes indépendantes, qui travaillent comme servantes ou prostituées, elle n'en fait en rien un état enviable.

Entre alors en lice en 1516, le personnage spectaculaire de Marfisa dans Orlando furioso de l'Arioste (la version définitive paraîtra en 1532), demoiselle revêtue d'une armure, qui combat avec fougue et sait aussi bien chevaucher que manier la lance et le bouclier. Dans un discours célèbre du Chant 26, cette puissante amazone des temps modernes (que le narrateur compare d'ailleurs à Penthasilée devant Troie) déclare fièrement à un chevalier Sarrasin, qui la veut comme butin de guerre, qu' elle n'appartient à personne d'autre qu'elle-même et n'a pas besoin de champion pour assurer sa défense ${ }^{17}$. Virginia Cox, qui a étudié l'impact de cette virago comme 
source d'inspiration de certains personnages du dialogue $I l$ merito delle donne de Moderata Fonte (1600), estime que cette combattante offre, au sein de la littérature du seizième siècle, la représentation très rare, sinon unique, d'une femme engagée à vivre une vie indépendante des hommes ${ }^{18}$. Même si cette remarque appelle d'ores et déjà quelques nuances pour ce qui est du caractère unique de cette représentation, elle demeure précieuse, car en donnant un prédécesseur littéraire au personnage indépendant de la Première fille de Marguerite qui assure sa défense à sa manière, il devient possible d'établir quelques éléments de continuité et de discontinuité dans l'histoire de la défense et illustration de l'émancipation de la jeune fille qui aspire à l'indépendance.

À la Renaissance, les humanistes lèvent le mépris entourant le mariage par rapport au célibat consacré et la société continue à critiquer les mariages de raison. Ceci explique que Saulnier voit dans le personnage de la Première fille « le droit, au départ de choisir son état, acceptant ou refusant l'amour (ici sans doute, une critique latente des mariages imposés) $\gg 19$.

Dans ce contexte de réévaluation des états de vie laïques et du droit au libre choix, la possibilité du célibat laïque féminin apparaît chez Érasme, dans les deux colloques de 1523 « La vierge qui hait le mariage » et « La vierge repentante », où il souligne que la maison familiale est un endroit de piété aussi pur et convenable que le couvent sinon davantage, vu les mœurs de certains ${ }^{20}$. Ainsi, à Catherine, résolue à rester vierge, son ami Eubule lui conseille dans « La vierge qui hait le mariage », la vie mariée qui conviendrait mieux à sa personnalité plutôt que le couvent en lui faisant observer que

C'est une chose magnifique que la virginité, si elle est sans tache, mais il n'est nullement indispensable que pour la conserver que tu te voues à une communauté dont par la suite tu ne pourras être retirée. Il t'est possible de garder ta virginité auprès de tes parents. ${ }^{21}$

Quoique le célibat ne soit pas envisagé comme un état permanent dans ces dialogues scolaires, la même dignité est néanmoins reconnue au célibat consacré qu'au célibat laïque ${ }^{22}$ tant pour la jeune fille que pour le jeune homme. Cette réévaluation se limite pour l'instant à choisir le lieu (le couvent ou la maison) ainsi que l'état (le célibat religieux ou le célibat laïque) le plus propice à la piété.

Il convient également de considérer l'exemple de l'abbaye de Thélème de Rabelais qui vient à sa façon faire progresser l'idée d'accorder plus de liberté à la jeune fille. Car, même s'il s'agit d'une utopie aristocratique, jeunes filles et jeunes hommes vivent, dans une abbaye mixte laïque, un espace de liberté en tout bien et tout honneur jusqu'à ce qu'ils quittent le couvent au moment de se marier. Pour Cioranescu, c'est le premier modèle 
de bonheur à ne plus dépendre des obligations de la foi et à rendre les Thélémites responsables de leur bonheur humain à l'instar de l'Éthique à Nicomaque d'Aristote ${ }^{23}$.

\section{La défense du « digne ranc » de la Première fille}

En 1542, Marguerite de Navarre met en scène un personnage de jeune fille, heureuse de le demeurer ${ }^{24}$. Celle-ci ne demande pas l'autorisation de choisir la vie de célibataire, mais l'élit de manière délibérée dans l'esprit aristotélicien, loin du modèle de soumission que l'on attend des filles. Outre cela, elle n'aspire ni à l'idéal de vierge et de martyre, pas plus qu'à celui d'intellectuelle ou de figure d'autorité politique ou militaire d'une collectivité.

Cependant, en contrepartie de son désir et de sa volonté de rompre avec la tradition, elle se sent tenue de faire la preuve de la dignitas de son état, c'est-à-dire de sa vertu. Ainsi, après avoir clamé le plaisir et le contentement de « liberté de corps » (v. 3), elle affirme bien haut pour rassurer la compagnie, qu'

Il n'y ha nul, qui par solicitude

Me sceust jamais oster ce digne ranc. (v. 9-10)

Voici écarté tout soupçon d'hédonisme, il n'empêche que l'intéressée va insister pour dissiper tout doute sur ses intentions honnêtes. Le troisième livre du Courtisan avait amplement montré quel jeu de faux semblants pouvait entourer les propos d'amour, comportement que L'Amye de court de La Borderie épinglera de façon provocante ${ }^{25}$. La Première fille de la Comédie choisit sans hésiter son camp, qui n'est pas celui des fausses apparences :

J'ayme verité :

J'ayme pureté

De cœur, et de corps.

Passion, Amour,

N'y fait nul sejour :

Je les metz dehors. (v. 292-97)

Aussi, quand le débat entre les deux filles reprend à la scène 4, à la suite de la longue plainte de la deuxième femme qui évoque le thème de la mal mariée, la Première fille est-elle renforcée dans son désir de maintenir

Liberté honneste

A garder, suis preste,

Sans m'en divertir. (v. 262-64) 
Cette idée de « liberté honneste » évoque à sa façon la devise « Fais ce que vouldras » de l'abbaye de Thélème, qui n'est pas synonyme de vie dissolue, mais de vie guidée par l'honneur ${ }^{26}$. Cependant, à l'encontre des Thélémites, déjà mariés ou qui le deviendront à leur sortie, ayant vécu « en dévotion et amytié », la Première fille exclut, de son éthique individuelle, l'amour et le mariage.

Toujours par besoin de se justifier, elle rejette fièrement tant le code de l'amour courtois purus avec ses «guerdons » et son serment de loyauté amoureuse que l'amour vénal dont elle se défend :

Mon coeur est à moy.

Je n'ay mis ma Foy

En don, ny en vente. (v. 277-79)

Ces paroles donnent une mesure de la distance qui sépare la Première fille du comportement ambivalent de sa contemporaine l'Amye de court qui accepte les dons et les cadeaux tout en prétendant ne rien donner en retour. Distance aussi de l'ancien idéal courtois christianisé, que résumait le vers célèbre de Cligès de Chrétien de Troyes «Qui a le cuer, cil a le cors »27, qui tentait de concilier fin' amor et mariage. En fait, c'est l'exact contraire que réclame la Première fille en déclarant vouloir s'appartenir entièrement (v. 7-8) plutôt que de donner son cœur et son corps à un mari. Distance également de l'exaltation de l'amour comme souverain bien de la Seconde fille et de son chaste plaisir néo-platonisant, véritable paradis sur terre (v. $376-87)^{28}$, que lui procure la présence de son ami.

La Première fille donne une fin de non recevoir, et pour toujours, à tous ces modèles amoureux, anciens ou contemporains, honnêtes ou non, par crainte de perdre sa liberté, car

Liberté garder

Veux sans m'hazarder

De jamais aymer. (v. 334-36)

Et sans doute, par mépris stoïcien pour ce qui est sensibilité, elle se met à chanter pour faire diversion aux pleurs et aux cris des amoureux en proie à la mélancolie érotique :

Les Amoureux laisse

Sans point les hanter.

S'ilz pleurent, ou prient,

Tant plus fort ilz crient,

Me prens à chanter. (v. 323-27) 
Il reste que tout est spéculation dans sa liberté, si sage à ses yeux. La Première fille, sans projet précis, ne sait pas encore ce qu'elle fera de cette liberté. En tout cas, ce n'est ni par souci de piété ni pour réaliser un projet profane comme celui de Catherine Des Roches qui refusera de se marier par choix quelques décennies plus tard ${ }^{29}$. De toute manière, la comédie qui nous occupe n'est pas le lieu où s'inquiéter de la suite des évènements ou des moyens concrets avec lesquels réaliser son rêve d'indépendance. Tout reste donc au niveau de la déclaration d'intentions.

Pour ramener la Première fille à la raison, ou plutôt à la tradition, la Vieille lui sert un discours en trois mouvements. En effet, le refus d'obtempérer à l'ordre naturel du puissant dieu Amour lui fait risquer une punition d'Amour, c'est-à-dire d'Anteros, le dieu vengeur de l'amour méprisé30 :

Amour est un fin, et faux Ange,

Qui trescruellement se venge

De ceux, qui de luy n'ont fait compte. (v. 540-42)

Puis, la Vieille cherche à amadouer cette adolescente encore inexpérimentée grâce au mythe explicatif de l'androgyne platonicien. Ainsi, la parfaite moitié qui lui est destinée ne lui serait pas encore apparue, suite à quoi naîtra le désir d'amour (v. 550-53).

Et, après ces concessions aux différents mythes d'Anteros et d'Éros de l'Antiquité païenne, la Vieille se souvient in extremis des ressources de la providence divine :

Ce sera la bonté haultaine,

Qui par le temps y pourvoyra. (v. 555-56)

La Vieille traite la Première fille comme on traite affectueusement et patiemment une adolescente qui veut refaire le monde. Ce spectacle de conflit de générations devait bien divertir l'auditoire. La Vieille, qui ne doute pas de sa vertu et de sa sincérité, lui prédit que l'Amour venant, elle finira par se ranger et ressentir les signes doux et amers de l'innamoramento qu'elle lui décline avec une pointe de plaisir nostalgique :

Jusques là l'on ne vous verra

Aymer : car vous estes trop fine,

Je le voy bien à vostre myne :

Car de rien ne faites semblant.

Amour, qui va les cœurs emblant,

Et le temps, qui doucement passe

Sans que vostre vertu s'efface

Vous feront changer de propos,

Trembler le cœur, battre les poux,

Et sentir le doux, et l'amer,

Que l'on peult souffrir pour aymer. (v. 557-67) 


\section{La Première fille de la Comédie et la Querelle des amies}

Replaçons maintenant le débat de la comédie des femmes dans l'actualité littéraire de 1542. La critique a vu dans la Comédie des quatre femmes la contribution de Marguerite de Navarre à la Querelle des amies ${ }^{31}$, lieu de débats littéraires pro et contra sur le milieu de la cour et, en fait, moins contre la cour qu'un certain comportement de la femme décrit dans Le livre du courtisan de 1528. Cette Querelle éclate en 1542 avec L'Amye de court qui trace le portrait satirique d'une jeune fille apparemment préoccupée avant tout par son plaisir, plus que sa vertu, avant de se ranger pour se marier ${ }^{32}$.

Luce Guillerm a certainement saisi l'ambiguïté ontologique qui entoure cette jeune fille en posant que «L'Amye est dotée d'un pouvoir de fascination qu'il ne faut pas dérober, une femme "libre", ni épouse, ni veuve, ni fille, ni bien sûr religieuse, ni putain, non plus, ni Célestine. [...] Hors statut précis, une errance qui est le péril même ${ }^{33}$. Position périlleuse qui expliquerait sans doute pourquoi la jeune protagoniste de L'Amye de court a donné lieu à des interprétations entièrement opposées. «Quasi putain » pour Michael Screech, l'Amye serait une adepte de l'évangélisme et de l'amor virtutis pour Cottrell ${ }^{34}$. Laissons de côté, pour l'instant, ces interprétations divergentes puisque la Première fille rejette à la fois Éros et Anteros, au sens christianisé d'honnête amitié qu'Anteros peut également prendre afin de comparer ces deux personnages par rapport à l'évolution des aspirations d'adolescentes.

Pour légitimer leurs revendications de liberté, l'Amye comme la Première fille usent de l'argument de nature, mais l'Amye y ajoutait une argumentation plus militante par rapport à la cause des femmes en déclarant que « ce présent céleste » de la liberté avait été usurpée par les hommes :

Cela provient qu'il est tout manifeste

La liberté estre présent céleste,

Que dieu voulut esgallement offrir

A tous vivans, dont ne povons souffrir

Qu'elle nous soit usurpée des hommes

Qui ne sont dieux, ny riens plus que nous sommes. (v. 411-15)

La Première fille, quant à elle, ne s'en prend pas aux hommes et ne se porte pas à la défense de toutes les femmes; n'invoque pas les dieux et ne se soumet pas à l'épreuve de la chasteté ${ }^{35}$. Plutôt, elle se contente de militer pour sa propre cause et de compatir avec les malheureux. Ce qui plus est, la Première fille refuse l'amour ainsi que ce jeu de la séduction trop risqué et ne partage pas une conception aussi ambiguë de la liberté de corps que celle de l'Amye. Cette dernière, malgré les assurances de la chasteté de son corps 
et de son esprit, appelle Éros « folie ou infelicité » (v. 22) et se fait une gloire de sa victoire sur Éros (v. 28), sans nous convaincre entièrement.

Cependant, c'est au niveau des finalités de leur agir qu'on peut les départager encore plus nettement. Pour l'Amye, la finalité de ces jeux est de trouver un mari, « le but de toute femme saige » (v. 758), ce qui lui fait dire à ce propos :

Et Premierement je mettray mon estude

Et emploiray peine \& solicitude

De le gaigner si bien qu'il m'aymera. (v. 809-10)

Et c'est ici qu' on a le sentiment d'entendre la Première fille répondre du tac au tac à l'Amye, dès le début de la Comédie, par ces vers :

Quant est de moy, j'ay mise mon estude

D'avoir le corps, et le cœur libre et franc. (v. 7-8)

Selon Danièle Trudeau ce personnage délibéré d'Amye, avec son antipétrarquisme et son anti-conformisme, donne "l'illusion d'entendre une voix non seulement "féminine", mais aussi "adolescente", ce qui est rare dans la littérature française de cette époque ${ }^{36}$. En tout cela, l'Amye et la Première fille se rejoignent de même que dans leur manque d'esprit de soumission et leur volonté de décider sans demander la permission à la famille ou à la société. Bref, toutes deux cherchent le repos de l'esprit et affichent la joie de la jeunesse, mais par des voies et pour des motifs opposés.

Au sens strict, la Première fille n'entre pas, à vrai dire, dans la Querelle des amies. Tout d'abord puisqu'elle ne recherche pas un ami, voire un mari, comme le fait l'Amye de court et, ensuite, parce qu'elle ne définit pas, non plus, son bonheur par rapport à la présence ou non de l'amour vertueux dans sa vie, à l'instar des autres personnages de la Comédie des quatre femmes. Par contre, en tant que personnage qui exalte le corps et le cœur libres en paroles comme en gestes, elle offre un contre-exemple, éclatant de droiture, à l'honnêteté plus douteuse de l'Amye. En ce sens, la Première fille apporte un élément de la réponse de Marguerite de Navarre à la Querelle des amies et sert à défendre sa conception élevée de l'honneur féminin.

Que représente, enfin, cette Première fille ? Nouvelle émule de la déesse Diane, soucieuse de garder sa chasteté et à l'affût d'un certain pouvoir? Une adepte de quelques idées stoïciennes, toutes profanes, sur la liberté inaliénable comme gage de bonheur ? Il y a plus, à notre avis. Ce personnage manifeste la possibilité de remettre en question le statu quo de l'échelle des valeurs féminines en plaçant la liberté au-dessus de la chasteté, tout en ne renonçant pas à la vertu. En d'autres termes, la chasteté n'est plus le but en soi, la vertu féminine par excellence qu'en a fait la doxa tant 
religieuse que sociale jusque là, mais plutôt un moyen servant sa liberté, revendiquée aux fins d'un bonheur individuel et non collectif. Et si recherche de pouvoir il y a, la Première fille ne réclame que de l'exercer sur elle-même.

\section{Du discours pro domo de la Première fille aux revendications collectives en faveur du célibat volontaire}

En tout cas, c'est une revendication semblable qui continuera à se faire entendre, de manière de plus en plus précise et argumentée, dans la fiction comme dans les réalités du temps, même si elle ne se fait que par petites touches, glissées ici et là, avant que ne paraisse l'audacieux manifeste de 1700 de Gabrielle Suchon Du célibat volontaire ou la vie sans engagement ${ }^{37}$.

En attendant, le personnage de Roseline dans la Bergerie (1583) de Catherine Des Roches invitera celui de Pensée, qui ne valorise pas la figure de l'amant martyr pétrarquiste, à chanter avec elle « la liberté des Dames », passage que l'éditrice Anne Larsen rapproche d'ailleurs, non sans raison, de la Comédie des quatre femmes de Marguerite de Navarre ${ }^{38}$. L'essentiel demeure de comprendre le sens de la chasteté chez Catherine des Roches, ce que fait Tilde Sankovitch avec beaucoup de perspicacité dans une étude mythopoétique sur le désir des femmes d'écrire et leur appropriation de figures mythologiques pour exprimer leurs quêtes. Dans le chapitre consacré aux Dames des Roches, celle-ci considère le désir de chasteté de Catherine moins sur le plan du statut sexuel qu'intellectuel et social, et moins comme stratégie de défense contre l'empiètement sur le corps que celui de l'esprit de la femme ${ }^{39}$.

Pour sa part, Madeleine Des Roches, influencée par la morale stoïque du culte de la volonté et du mépris des choses fortuites, fera valoir dans un sonnet des Secondes ceuvres, de 1583, que le bonheur est possible dans le célibat, sans dénigrer pour autant celui des genres de vie traditionnels du mariage et du veuvage, qui passent par l'amour conjugal ou son souvenir après sa perte. Une coloration aristotélicienne est également perceptible dans ces vers qui font de chacun (et de chacune, en l'occurrence) le responsable de son bonheur humain ${ }^{40}$ :

Je croy que le bonheur ne dépend que de nous

[...]

Celle qui a de l'heur sans estre mariée,

Elle est heureuse aussi en se trouvant liée

Aux saintes loix d'Hymen, et si amour l'esprit

Avec l'heureux flambeau d'un chaste mariage, Elle est heureuse encor en son simple veufvage, Pource que son bonheur depend de son Esprit ${ }^{41}$. 
L'idée du célibat volontaire n'agite pas que les esprits en France, aussi deux œuvres, l'une italienne, l'autre espagnole, méritent d'être au moins signalées pour leur convergence avec l'argumentation avancée jusqu'ici et leur clairvoyante sensibilité à l'aspect économique de la question.

En effet, le dialogue Il merito delle donne (1600) de Moderata Fonte s'exprime de manière encore plus affirmée sur le célibat volontaire, même s'il ne s'agit pas de la revendication centrale de ce débat d'une grande franchise entre sept aristocrates vénitiennes réunies chez la veuve Leonora. Virginia Cox reconnaît dans l'un des sonnets du personnage de la savante Corinna un véritable manifeste en faveur du célibat volontaire. Caractère mis en valeur, selon l'éditrice, par le deuxième vers du premier quatrain qui paraphrase les fières paroles de la Marfisa de l'Orlando furioso : «Libre est le cœur qui séjourne dans ma poitrine, Je ne sers personne, et n'appartiens à personne d'autre que moi-même. La modestie et la courtoisie sont mon pain quotidien; La vertu m'exalte et la chasteté me pare ${ }^{42}$. On retrouve au sein de ces vers la même exaltation qui enfièvre les répliques de Première fille de Marguerite de Navarre, d'autant plus que toutes deux accordent la préséance à la liberté du cœur et du corps plutôt qu'à la chasteté, introduite en tant que moyen et non fin en soi ${ }^{43}$. Autour de Corinna, on vante le bonheur de sa liberté par rapport à celui des trois âges de la femme en faisant l'éloge des avantages propres au célibat, dont celui de la liberté de s'instruire et de se cultiver, y compris celui d'écrire et d'assurer à son auteur gloire céleste et terrestre ${ }^{44}$. En fait, de manière très lucide et sans complaisance, ces femmes mettent le doigt sur le problème des vénitiennes d'alors, qui est non seulement celui des principes abstraits et élevés de la liberté et du bonheur, mais celui de la question économique. En effet, les systèmes de la dot, du droit à la propriété et des héritages constituent, le plus souvent, des entraves à leur liberté puisque celles-ci sont, sauf exception, privées de la gestion de leurs biens.

Toujours dans la fiction, le désir de rester libre et vertueuse du personnage de Marcelle, la belle bergère, dans Don Quichotte de la Manche (1605), reste largement incompris autour d'elle où d'aucuns la tiennent responsable de la mort son admirateur Chrysostome. Le discours qu'elle livre à cette occasion fait état des motifs de sa volonté de vivre sans serviteur amoureux, de son double refus d'Éros comme d'Anteros, de sa soif inaltérable de liberté. Fait plus rare, la question des moyens financiers qui rendent possible son indépendance et sa vie dans la sereine solitude, au milieu de la nature, en compagnie des arbres est abordée, sans ambages ${ }^{45}$.

Quelques années plus tard, les reproches se précisent dans Le grief des dames (1626) de Marie de Gournay où l'ironie grinçante n'atténue pas la poignante gravité de son incipit célèbre. Celle-ci dénonce les horizons 
limités offerts non à une femme en particulier, mais au sexe féminin qui doit se contenter de vertus qui n'en sont pas et d'un bonheur étriqué d'où la liberté, plus particulièrement celle de s'instruire et d'être indépendante, est interdite ${ }^{46}$. Sur le plan personnel, Marie de Gournay porta avec fierté le titre de damoiselle, même si pour cela, il lui fallut résister aux pressions de sa mère pour la marier, vivre petitement, et plus tard, se voir tournée en dérision pour avoir voulu « prétendre à l'indépendance économique en vivant de sa plume, et seule, par choix $\gg^{47}$.

En 1693, Gabrielle Suchon publie son Traité de la morale et de la politique dont les chapitres sous forme de diptyques commencent par traiter de l'essence de la liberté qui vient de Dieu (c'est aussi ce que faisaient valoir l'Amye de Court et la Première fille), puis des différentes sortes de liberté selon les grands philosophes, tout particulièrement Aristote, Sénèque et saint Thomas d'Aquin pour décrire, une fois ces grands principes établis, la réalité de la privation de ces biens dont souffrent les femmes ${ }^{48}$. L'auteur, qui est une religieuse défroquée, déplore, par exemple, la privation de la liberté de voyager qui empêche d'apprécier la diversité des créations de Dieu en invoquant l'argument de nature (comme l'Amye de Court et la Première fille) selon lequel même les oiseaux changent de région, mais étoffe son argumentation de chapitres riches des exempla des pèlerinages des saintes du christianisme primitif ${ }^{4}$ 9. Gabrielle Suchon lie aussi et liberté et bonheur, biens dont l'absence condamne femmes et filles au malheur, ce qui la rapproche de ses prédécesseurs ${ }^{50}$. La principale revendication de ce traité, cependant, porte sur le libre choix des états de mariage et de religion, ce qui amène l'auteur à suggérer la possibilité d'une troisième voie.

C'est cette troisième voie, celle de la vie neutre, de la vie dégagée par rapport au mariage ou à la vie du cloître, aussi appelée la vie heureuse et la vie désirable qui fait l'objet du livre Du célibat volontaire ou la vie sans engagement en 1700. Pour être une parfaite « Neutraliste », le célibat volontaire doit être choisi de propos délibéré et de volonté ferme et non par calcul $^{51}$. L'auteur prend soin d'écarter le libertinage de cette notion de liberté, car, sans vouloir amoindrir les autres états, elle tient surtout à en défendre la valeur morale, voire la sainteté. Ce manifeste allègue les douze avantages de cet état, pour faire ressortir ce qui le rapproche et le distingue des autres états, entre autres, la possibilité de la «fécondité morale et civile » plutôt que naturelle qu'offre le fait de s'occuper des pauvres en lieu d'enfants. L'auteur reconnaissant sans ambages la hardiesse de ce choix en raison des effrontés et des médisants du monde devant ce genre de vie qui rebute même les parents de celles qui désirent garder la qualité de Fille.

Et lorsque Gabrielle Suchon déclare que «C'est un titre fort honorable que celui d'honnête fille, car une conduite sage et vertueuse met une 
personne dans le premier de tous les rangs ${ }^{52}$ et qu' elle consacre un chapitre entier à en détailler le bonheur tranquille, à mi-chemin entre le mépris stoïcien pour les choses fortuites et la charité chrétienne aux nécessiteux, on est ramené, au-delà de l'argumentation copieuse et soutenue de son discours étayé par les autorités profanes et sacrées, à ce "digne ranc » dont s'enorgueillissait la Première fille de la Comédie des quatre femmes et à ce « repos » dans lequel elle se plaisait.

\section{Conclusion}

Terminons en disant que, dans cette comédie, Marguerite n'érige pas en modèle pour toutes ce personnage de la Première fille unique dans son œuvre pas plus que celui de la tout aussi vertueuse Seconde fille d'ailleurs qui ne vit que pour l'amour de son ami. Dans cette pièce jouée dans l'ambiance de fête des jours précédant le Carême, Marguerite a, certes, accusé les traits de chacune de ces jeunes filles dans une intention dramatique, mais il reste qu'elle les a inventées avec leurs excès et que chacune de ces exaltées reflète une part de réalité et une part de rêve des modèles de bonheur pour une jeune fille. L'auteur a surtout voulu défendre la dignité du choix de chacune.

Et quand bien même la Seconde fille récuserait les idées de la Première avec élan et que la Vieille ne prendrait guère au sérieux ces rêves de «jeune fille », trop irréalistes à ses yeux, le personnage novateur de la Première fille ouvre la voie à une vision nouvelle du célibat féminin, non plus perçu comme un mode de vie scandaleux ou méprisable, mais comme une source possible de bonheur et de vertu.

Ce nouveau modèle féminin de félicité à la Renaissance, qui ne porte pas un masque de vertu comme le personnage de l'Amye en attendant de trouver mari, ne veut rien devoir à Éros. Il ne relève pas, pour autant, exclusivement du courant antérotique, au sens de refus de l'Amour, car cette vision restrictive distrairait du chamboulement et du réaménagement de valeurs, sous nos yeux, en faveur de la liberté et du désir de réalisation personnelle. Certes, Simone de Beauvoir récusera le lien de cause à effet entre liberté et bonheur féminin, dès les premières pages du Deuxième sexe $(1949)^{53}$, mais ne fallait-il d'abord faire l'expérience de cette valeur qui donne un but plus exaltant que celui de la défense acharnée de la chasteté comme unique vertu et unique mérite ?

Il apparaît désormais que la Première fille de Marguerite n'avance pas complètement seule dans l'histoire de l'émancipation de la jeune fille indépendante. Si tous s'entendent pour parler de la rareté du personnage de la jeune fille dans la littérature, la catégorie de celles qui ne choisissent ni le mariage ni la vie conventuelle est encore plus limitée dans la littérature, 
alors que le phénomène démographique lui va en augmentant au XVI ${ }^{\mathrm{e}}$ siècle tout comme les revendications en faveur de ce genre de vie qui se multiplient et se font entendre avec plus d'insistance dans le dernier quart de ce siècle. Petit à petit, on observe les plaidoyers pro domo du célibat librement consenti (la Première fille, Marcelle) se transformer en revendications collectives, du moins en théorie, car en pratique, cette possibilité n'est accessible qu'à celles qui disposent de moyens financiers pour assurer leur autonomie (Fonte, Gournay, Cervantès, Suchon). Si certaines figures historiques et littéraires comme Jeanne d'Arc, Élisabeth Ire et Marfisa sont marquées par l'androgynie, celles qui n'aspirent pas à un rôle de premier plan dans la vie publique ou militaire ne cherchent pas à estomper leur nature de femme que cela soit de manière vestimentaire ou autre.

Surtout, comme ces discours ne sont pas tous directement tributaires les uns des autres, malgré des filiations avérées entre l'Arioste et Moderata Fonte, puis Olympia Morata et Catherine des Roches, ainsi que celle plus diffuse d'Érasme sur les esprits, certaines constantes n'en sont que plus frappantes. Ainsi, malgré des différences de ton, telle l'insouciance des personnages adolescents, qui veulent tout, tout de suite, tandis qu'une Gabrielle Suchon voit la situation avec le recul et la sérénité du haut de ses soixante ans passés, tous ces textes sont sensibles au fait d'aller contre la coutume et l'opinion. D'ailleurs, dans tous ces textes, se font entendre les réactions négatives de l'entourage, malgré les protestations répétées de vertu des protagonistes, à travers les rires, la suspicion généralisée, les railleries, les critiques méprisantes quand ce ne sont pas les médisances et les calomnies ou pire, le refus catégorique de leurs aspirations que la doxa désapprouve et que l'insidieuse immuabilité des lois défavorise (I. Nogarola, la Première fille de M. de Navarre, O. Morata, Catherine Des Roches, Marcelle de Cervantès, Mlle de Gournay, G. Suchon). Il ne faut pas oublier non plus les sévères traités de morale, rédigés à leur intention, tel celui de Villethierry (voir la note 49).

Ce contexte social, à l'avance défavorable, oblige celles qui ne choisissent ni le mariage ni le cloître, dans les realia comme la fiction, à mener plusieurs combats à la fois. Le premier est de faire reconnaître cette catégorie sociale en tant que telle par rapport aux autres genres de vie, puis d'en faire valoir ses mérites propres, tout particulièrement la valeur positive de cette vie, source de vertu et de bonheur. La nette volonté d'en faire une valeur positive s'exprime par la profession publique d'attachement à la vertu de chasteté, envisagée comme moyen privilégié, mais nulle part comme le but de la vie vertueuse. Il leur faut aussi se défendre des accusations sourdes de choisir le célibat laïque par pur égoïsme en arguant de l'utilité de cette vie. L'argumentation sur la moralité et l'utilité de leur choix s'accompagne de l'argu- 
ment de nature de la liberté, parfois de notes humoristiques ironiques, et très souvent d'exempla livresques mythologiques et historiques, dont quelquesuns sont contemporains et personnels, ce qui leur fournit également des modèles pour se construire et des raisons d'espérer. Dans les textes plus tardifs, les mentions et les débats relatifs à l'argent (Fonte, Gournay, Cervantès) reflètent le malaise d'une société qui reconnaît aux femmes des biens nominalement, mais leur en restreint la gestion. Le texte de Moderata Fonte s'en prend particulièrement aux hommes, détaillant les torts, le cas échéant, des pères, frères, maris, amants qui entravent les désirs de liberté des femmes alors que d'autres, comme celui de Marguerite, embrasse la liberté sui generis, sans égard aux comportements masculins.

La liberté, pour quoi faire ? Pour être tout simplement, s'appartenir cœur et corps réunis, choisir son genre de vie, le droit à aimer ou non, posséder ce bien inaliénable qu'est la liberté. La liberté du célibat volontaire leur semble conduire à cette liberté spécifique qui est celle de l'accès à la culture et, dans plusieurs cas, à l'écriture (Nogarola, Des Roches, Gournay, Fonte) de telle sorte que de leur solitude studieuse d'où a été volontairement écarté le modèle de bonheur qui passe par Éros, elles ne ressentent pas le vide intérieur que s'imagine l'opinion reçue, mais plutôt un sentiment de plénitude issu des joies intellectuelles de la création à laquelle préside la Vénus céleste. Les textes révèlent aussi le désir de plus en plus vif de jouer un rôle dans la cité, et tout particulièrement auprès de leurs consœurs, en se rendant utiles de bien des façons (Fonte, Suchon), par le soin de leurs parents âgés ou bien des enfants nécessiteux des autres qu'elles se proposent d'élever ou d'instruire.

À l'encontre des idées reçues sur le célibat volontaire qui en font une source de malheur, les personnages de papier ou historiques rencontrés se présentent bien droits, respirant la joie, intimement convaincus de la dignité de leur état, malgré les embûches sans nombre sur leur route. D'où ces fréquents éloges du bonheur qui parsèment ces quelques textes, où il $\mathrm{y}$ a une nette propension à emprunter les conceptions du modèle du bonheur néostoïcien (M. de Navarre, C. des Roches, G. Suchon), dont le repos tranquille fait bon ménage avec les vertus chrétiennes de charité et de compassion à l'endroit des autres. Outre cela, la place reconnue au bonheur humain n'est pas incompatible avec le bonheur céleste, chez la jeune fille mise en scène par Érasme ainsi que la Corinna de Fonte et la Marcelle de Cervantès, il en était même le prélude chez Nogarola.

Ainsi, sous ces dehors de comédie profane, la Comédie des quatre femmes, n'en apporte pas moins, au milieu des rires et de la bonne humeur, sa contribution à la délimitation d'un terrain d'essai propice, dans l'imaginaire spéculatif 54 à l'amorce d'un changement des mentalités. Chez la 
Première fille, la liberté de façonner son destin dans ce nouveau territoire « hors statut précis » 55 en est encore à ses balbutiements, même si tous les arguments essentiels y sont déjà, hormis les autorités philosophiques et religieuses et les exempla. Et ce sont justement ces touches éparses, ces envols gonflés d'une sève nouvelle vivifiante qu'il importe de ne pas effacer de la mémoire collective.

\section{Université du Québec à Montréal}

\section{Notes}

1. La recherche pour cet article a été menée avec l'appui du CRSH (Ottawa) que nous remercions tout comme Mesdames Olga Anna Duhl et Rebecca Hayward pour leurs utiles suggestions.

2. Marguerite de Navarre, Comédie des quatre femmes (sous le titre de Comédie à dix personnages), in Théâtre profane, éd. V.-L. Saulnier, nouvelle éd., Genève, Droz, Paris, Minard, 1978, p. 83-126, édition citée dans cet article. Voir aussi l'édition récente de la Comédie des quatre femmes in Marguerite de Navarre, Euvres complètes, t. 4, (Théâtre), éd. Geneviève Hasenohr et Olivier Millet, Paris, H. Champion, 2002, p. 365-407 et p. 367 pour sa désignation comme momerie de carnaval.

3. Les genres de vie les plus connus sont décrits dans La République de Platon et l'Éthique à Nicomaque d'Aristote, modèles qui furent remaniés et remodelés par les épicuriens et les stoïciens selon leurs propres échelles de valeurs ; à ce sujet, voir Robert Joly, Le thème philosophique des genres de vie dans l'Antiquité classique, coll. "Classe de Lettres et de Sciences morales et politiques », t. 51, fasc. 3, Bruxelles, Palais des Académies, 1956.

Pour une esquisse de la fortune de ce thème au Moyen Âge et à la Renaissance, thème qui entre en concurrence avec la vision chrétienne du bonheur dans l'au-delà, voir nos " Prolégomènes à une histoire de la félicité ", in Portraits du bonheur au Moyen Âge et à la Renaissance. Douze modèles de félicité céleste et terrestre, numéro spécial de Memini publié sous notre direction, $\mathrm{n}^{\mathrm{O}} 6,2002$, p. 5-58.

4. Fulvia de Luise et Giuseppe Farinetti, Storia della felicità. Gli antichi e i moderni, coll. « Piccola Bibliotheca Einaudi », nouvelle série Filosofia, no 110, 2001, Turin, Einaudi, p. XVI : «La felicità filosofica, nella quasi totalità delle sue varianti, è una proposta esclusivamente al maschile. L'uomo ideale non è mai una donna e tutto ciò che gli si riconosce come soggetto attivo e consapevole, protagonista della sua vita e titolare dei suoi desideri, spinge nell'ombra il femminile, la passiva, paziente, accogliente presenza delle donne sullo sfondo. Il loro silenzio pesa sull'immaginario della felicità. [...] sono uomini che parlano a uomini, senza uscire mai da se stessi. Un' altra storia della felicità potrebbe essere scritta. » (nous traduisons).

5. L'étude de Marie-Thérèse Lorcin établit que Christine de Pizan est la première à ne pas se limiter à la «traditionnelle division tripartite adoptée par les clercs des trois âges de la femme (vierge, mariée, veuve) » pour décrire la population féminine en faisant aussi appel à « des facteurs économiques, sociaux et démographiques » (Marie-Thérèse Lorcin, "Le Livre des Trois Vertus et le Sermo ad status », in Une femme de lettres au Moyen Âge. Études autour de Christine de Pizan, éd. Liliane Dulac et Bernard Ribémont, coll. « Medievalia », no 16, série «Études christiniennes », Orléans, Paradigme, 1995, p. 139-49 et p. 148 pour les citations). 
6. Pour les critères de Christine de Pizan, voir Marie-Thérèse Lorcin, « Christine de Pizan analyste de la société », in The City of Scholars: New Approaches to Christine de Pizan, éd. Margarete Zimmermann et Dina De Rentiis, coll. «European Culture Studies in Literature and the Arts », Berlin, Walter de Gruyter, 1954, p. 197-205 et p. 204 pour la citation. Quant aux possibilités de bonheur humain de Christine elle-même, Claire Le Brun-Gouanvic rend compte de l'expérience de la vie contemplative (ou intellectuelle) du monde des livres qui lui procure une felicità mentale, dans son article, « Le lexique de la felicitas chez Christine de Pizan ", in Portraits du bonheur au Moyen Âge et à la Renaissance. Douze modèles de félicité céleste et terrestre, éd. cit., 2002, p. 103-27.

7. Daniel Ménager, « Modèles de vie », in Précis de littérature française du XVIe siècle, éd. Robert Aulotte, Paris, Presses Universitaires de France, 1991, p. 326-31.

8. Pour ce constat, voir Madeleine Lazard, Les avenues de Fémynie. Les femmes et la Renaissance, Paris, Fayard, 2001, ch. 3, p. 38 : «En un siècle où la fille n'a d'autre alternative que le mariage ou le couvent, quelle que soit sa condition, l'union conjugale est son destin, sa "profession" obligée ». Par ailleurs, on ne peut passer sous silence la possibilité d'offrir un espace de liberté en réaction aux contraintes du mariage qui existait déjà parfois à l'intérieur du célibat consacré ; voir Julie Hassell, Choosing Not to Marry: Women and Autonomy in the Katherine Group, London, Routledge, 2002, p. 137. Celle-ci examine un ensemble atypique de cinq vies du début du XIII ${ }^{\mathrm{e}}$ siècle, dont celles des vierges et martyrs Catherine, Marguerite et Juliana, où l'insistance n'est pas sur les bénéfices sacrés de la virginité, telle l'union mystique, mais sur les avantages procurés par le refus du mariage pour la détermination de soi.

9. Notre traduction de « Social life did not include a category for the unmarried woman outside of the religious life ; the women who chose that route inhabited an uncomfortable limbo », tiré de Margaret L. King, Women of the Renaissance, Chicago, University of Chicago Press, 1991, p. 28.

10. Signalons deux articles éclairants de Marcel Bernos, bien que portant sur le XVII ${ }^{\mathrm{e}}$ siècle. En effet, le premier «La jeune fille à l'époque classique », in De la pucelle à la minette. Les jeunes filles de l'époque classique à nos jours, éd. Marcel Bernos, Éliane Richard, Yvonne Knibiehler et Élisabeth Ravoux-Rallo, Paris, Messidor/Temps actuels, 1983, p. 13-69, fait remonter les origines de certaines attitudes de la famille et de la société à l'égard de la jeune fille jusqu'au XIII ${ }^{\mathrm{e}}$ siècle, procède à l'examen philologique du terme complexe de « jeune fille » et de termes reliés, tel «pucelle», s'interroge sur la frontière entre « jeune fille » et « vieille fille », fait état de la notion de puberté qui arrive plus tard que de nos jours et note l'absence encore au XVII ${ }^{\mathrm{e}}$ du féminin d' «adolescent» (p. 14-17). Aussi, plusieurs observations pourraient s'appliquer déjà à la condition de la jeune fille à la Renaissance, tout spécialement ce constat que «Les jeunes filles n'ont donc pas d'existence en elles-mêmes, pour elles-mêmes ; ce que va confirmer l'étude successive de leurs deux "vocations" : le mariage et la vie religieuse. Et paradoxalement, il est exceptionnel, au moins avant le $18^{\mathrm{e}}$ siècle, que des femmes remettent en question le système. Le mariage est généralement accepté, hormis par les Précieuses, comme la base de la société ; même par des "féministes" comme Mmes Des Roches dans le troisième quart du $16^{\mathrm{e}}$ siècle » (p. 41). L'article examine aussi le sort peu enviable des filles pauvres et abandonnées, reléguées au travail peu rémunéré des ateliers ou de la domesticité. Enfin, l'auteur estime avec pertinence (p. 28 et sq.) que la double tradition à l'égard de la femme, tradition issue du Moyen Âge, qui la voit tantôt selon une perspective gauloise, tantôt idéalisante, se répercute encore au XVII ${ }^{\mathrm{e}}$ siècle dans l'idée qu'on se fait de la jeune fille. Objet de tentation pour les religieux, tel Villethierry, elle suscite le respect, chez plusieurs moralistes et pédagogues, tel Fleury. Dans ses « Regards complémentaires. La jeune fille en France à l'époque 
classique », Clio. Histoire, Femmes et Sociétés, no 4, 1996, p. 161-65, Marcel Bernos reconnaît malgré ces difficultés terminologiques que «La jeune fille n'en existe pas moins pourtant en Occident : elle s'est épanouie depuis le Moyen Âge grâce au retard de l'âge au mariage, qui permet à une personne de sexe féminin, sortie de l'enfance, mais non encore insérée dans la société par le mariage précisément, d'avoir une portion de vie peu ou prou autonome » (p. 161-62). Et de rappeler que cette possibilité concerne les classes les plus élevées de la société. Voir aussi la note 13 infra pour une étude de synthèse sur l'aspect démographique de cette question.

11. Pour cette dernière, voir Philippa Berry, Of Chastity and Power: Elizabethan Literature and the Unmarried Queen, London, Routledge, 1989.

12. Après avoir fait état des humiliations injustifiées que dut subir I. Nogarola, car, selon d'aucuns, une érudition qui sortait de l'ordinaire devait couvrir une sexualité incestueuse contre-nature, Margaret King conclut qu' «Un chœur de voix rappela à Nogarola de préserver sa virginité intacte : car c'était pour une femme la référence par excellence à toutes ses occupations, y compris celle de solitude studieuse » (notre traduction de : «A chorus of voices reminded Nogarola to guard her virginity intact : it was the prime credential for a woman in all her pursuits, including that of studious solitude » [Women of the Renaissance, p. 195-98 et 198 pour la citation]).

13. Scarlett Beauvalet-Boutouyrie, «La femme seule à l'époque moderne : une Histoire qui reste à écrire », Annales de démographie historique, $\mathrm{n}^{\mathrm{O}}$ 2, 2000, p. 127-41 et p. 129 pour la citation. Ce bilan porte sur les recherches sur la catégorie sociale de la femme seule qui ne serait apparue comme un champ d'étude chez les historiens qu'à partir des années 1980. La notion de femme seule comprend ici l'état de célibataire (où sont distinguées les célibataires définitives, à partir de 50 ans, des temporaires en attente d'un mari) et celui de veuve. Ceci donne l'occasion d'observer que l'image et le statut de ces femmes sont déterminés par l'état matrimonial, car « contrairement au veuvage qui confère indépendance et autorité, le célibat ne donne pas à la femme le privilège d'être indépendante » (p. 131).

14. Ibid., p. 130. Notons que le célibat est considéré définitif dans ces statistiques à partir de 50 ans.

15. Ibid., p. 133, où il est aussi question des mesures adoptées par certaines autorités municipales du $\mathrm{XVI}^{\mathrm{e}}$ siècle pour contrôler les célibataires. Il est aussi fait allusion à la pratique mal connue de gardiens ou de tuteurs pour les filles ou veuves, sans père ou mari, possédant des propriétés, dans ce cas-ci dans la ville de Gand au XIVe siècle, dans Margaret King, Women of the Renaissance, p. 51.

Pour un rappel des craintes traditionnelles entourant une trop grande liberté d'action octroyée aux filles, voir les Élegies de la belle fille, lamentant sa virginité perdue avec plusieurs Épistres, Épigrammes, Instructions \& Traductions morales (1557), composées par Ferry Julyot, éd. E. Courbet, Paris, L. Willem, 1873. Cette jeune fille, devenue débauchée, raconte ses mésaventures et finit par reprocher à ses parents de l'avoir laissée trop libre : « Vous me deviez (pour vray) en mon jeune eage / Ainsi brider, car j'estoys trop volage» (p. 31). Toutefois, il est plus courant, dans les realia, d'entendre dénoncer les abus de l'autorité paternelle, soucieuse de la virginité de sa progéniture à marier, « joyau qu'il faut conserver à tout prix de peur d'être laissée pour compte », comme en témoigne l'étude de Gabriel A. Pérouse, Nouvelles françaises du XVI siècle. Images de la vie du temps, Genève, Droz, 1977, p. 290. Voir aussi le commentaire de Madeleine Lazard, Les avenues de Fémynie, selon lequel « [...] la vieille fille fait honte à sa famille et lui est une charge » (p. 38). 
16. Françoise Paradis, «La représentation de la jeune fille dans quelques romans du Lancelot-Graal », Perspectives médiévales, ${ }^{0} \mathrm{10}, 1984$, p. 85-87. Notons, au passage, un épisode d'environ un millier de vers, qui fait figure d'exception à cette tendance, dans Perceval de Chrétien de Troyes et sur lequel Jeanne Lods a attiré l'attention dans « La pucelle aux manches petites », in Mélanges offerts à Jeanne Wathelet-Willem, Liège, Cahiers de L'A.R.U.Lg, 1978, p. 357-79. Cette enfant d'une douzaine d'années, précocement amoureuse de Gauvain, passe en gestes et paroles de la naïve enfance au début de la maturité d'une demoiselle amoureuse. Ce passage est symbolisé par son insistance, qui se manifeste avec beaucoup de détermination et de liberté de langage, auprès de son père afin qu'il lui donne de larges manches, elle, dont l'âge ne lui donne droit qu'à de petites, afin de suivre la coutume des dames d'alors de donner une de leurs manches à celui qui combat pour elles.

17. Ludovico Ariosto, Orlando Furioso, trad. et éd. Guido Waldman, Oxford, Oxford University Press, 1974, p. 316.

18. Moderata Fonte (Modesta Pozzo), The Worth of Women: Wherein Is Clearly Revealed Their Nobility and Their Superiority to Men, éd. et trad. Virginia Cox, coll. «Other Voice in Early Modern Europe », Chicago, University of Chicago Press, 1997, p. 50, n. 8.

19. V.-L. Saulnier, éd. cit., p. 90. Ce thème fera les beaux jours de la comédie humaniste française où le comble du bonheur du personnage de la jeune fille bourgeoise sera non pas de rester célibataire, mais d'épouser le jeune homme de son choix après bien des péripéties ; voir à ce sujet, Madeleine Lazard, «Les félicités (très) terrestres des femmes dans quelques comédies de la Renaissance française », in Portraits du bonheur au Moyen Âge et à la Renaissance, loc. cit., p. 285-304.

20. Pour ces deux colloques voir Érasme, Euvres choisies, éd. Jacques Chomarat, Paris, Livre de Poche, 1991, p. 680-92 et 693-96 et p. 684 pour la citation.

21. Érasme, op. cit., p. 684. Dans le but de distinguer la morale de M. de Navarre de celle de Jeanne Flore à propos de la virginité, G. A. Pérouse, op. cit., se plaît à imaginer la réaction de Marguerite à la lecture de ce colloque : « [...] gageons que, lorsqu'elle lisait le colloque érasmien de la Vierge qui refuse le mariage, traduit par Marot, si la Reine approuvait pleinement la féroce satire des couvents, en revanche elle croyait possible, et très méritoire, la pieuse virginité suggérée par Érasme ; à Jeanne Flore, il semble que toute virginité ait dû paraître crime, ou tout au moins sottise » (p. 91). Plus sérieusement, l'auteur précise que ce colloque ne fut traduit par Marot qu'en 1549 « mais le thème, sinon le texte en était public depuis longtemps » (p. 91, n. 43).

Cependant, bien avant ce colloque d'Érasme, signalons que l'idée même que Cecilia Gonzaga (1425-51) vive sa pieuse virginité dans la riche maison paternelle avait paru peu réalisable à l'humaniste et théologien vénitien Gregorio Correr (1409-64). En effet, Cecilia qui voulait entrer au couvent à 18 ans, peut-être pour y poursuivre en paix ses études de femme humaniste sachant le grec et le latin, en fut empêchée par son père. Ce dernier accepta, pourtant, entre-temps, de renoncer au projet de mariage pour sa fille, à condition qu'elle reste à la cour et ne choisisse pas le cloître. Aux yeux de Correr, ce compromis paternel reste proprement incompréhensible comme il l'écrit dans une lettre de 1443 à Cecilia, car il la rend vulnérable au monde. En effet, en restant dans la maison paternelle, cette vierge s'expose aux dangers de la vie séculière, c'est-à-dire à l'attrait des chants et de la musique des banquets, des repas raffinés, de la mixité, voire à la vue de ses belles-sœurs enceintes, puis jeunes mères. Ne pouvant s'opposer à la volonté du père ni encourager Cecilia à s'enfuir, Correr lui propose de considérer sa pieuse mère comme son abbesse pour la conseiller sur la conduite et les études qui conviennent à celle qui renonce au monde dans le monde. Même si Cecilia et sa mère entrèrent au couvent à la mort du père en 1444, cet 
exemplum est très instructif sur les tensions entre les générations que suscitent la question du choix de vie ainsi que les craintes entourant la jeune fille laissée à elle-même pour garder sa vertu. Voir « Gregorio Correr to the Virgin Cecilia in Fleeing this Worldly Life », lettre 17 in Her Immaculate Hand: Selected Works by and about the Woman Humanists of Quattrocento Italy, éd. Margaret L. King et Albert Rabil, Jr., Binghamton, New York, Center for Medieval and Early Renaissance Studies, 1983, p. 91-105.

22. Ces colloques ne font pas non plus d'Érasme un champion de la liberté féminine à tous crans, puisqu'il verra d'un très mauvais œil les veuves prendre trop de plaisir grâce à leur liberté. Aussi, dans le sillage de saint Paul, il distingue les vraies des fausses veuves dans le maintien de leur dignité : voir « The Christian Widow », in Erasmus on Women, éd. Erika Rummel, Toronto, University of Toronto Press, 1996, p. 194.

23. Alexandre Cioranescu, «La littérature française et la recherche du bonheur », in La quête $d u$ bonheur et l'expression de la douleur dans la littérature et la pensée françaises. Mélanges offerts à Corrado Rosso, éd. C. Biondi, C. Imbroscio, M. J. Latil, N. Minerva, C. Pellandra, A. Sfragoro, B. Soubeyan et P. Vecchi, Genève, Droz, 1995, p. 23-24.

24. Il est piquant de remarquer que la même année Rabelais lance son Panurge dans une suite de consultations incluant jusqu'aux conseils du fou Triboulet pour savoir s'il devrait se marier ou non.

25. Le livre du courtisan brosse un tableau idéal de la cour et du courtisan, de la courtisane et de la façon de se gouverner en amour, où la façon platonique d'aimer, plus précisément celle du néo-platonisme ficinien, est valorisée malgré les mises en garde à l'endroit de l'amour déshonnête, vu le penchant qu'auraient certaines belles femmes à cumuler les amoureux comme «preuve de leur beauté »; voir Baldassar Castiglione, Le livre du courtisan, d'après la version de G. Chappuis (1580), éd. Alain Pons, Paris, Flammarion, 1991, p. 299. Dans L'Amye de court, tout se passe comme si l' auteur Bertrand de la Borderie avait été inspiré par l'idée de ce péché mignon décrit par Castiglione pour mettre en scène une jeune fille « libre, franche, coquette, presque cynique qui n'hésite pas à exploiter toutes les ressources du corps, une sorte de "capital érotique" et de l'esprit, pour affirmer son identité, pour obtenir le plaisir et la liberté de penser, de parler, de circuler. Le mariage est pour elle dans le VI ${ }^{\mathrm{e}}$ des VII mouvements du poème, une sorte de "retraite", [quand sa beauté sera passée] un choix où l'intérêt domine mais qui ne lui interdit pas de rêver une autre sorte de mariage parfait qu'elle croit mériter » (Henri Chamard, puis Rosanna Gorris, «La Borderie », in Dictionnaire des lettres françaises, Le seizième siècle, éd. Michel Simonin, Paris, Fayard, 2001, p. 656-57).

26. Ce passage célèbre se trouve dans François Rabelais, Gargantua, Euvres complètes, éd. Guy Demerson, coll. «L'intégrale », Paris, Seuil, 1973, ch. 57, p. 203 : «parce que gens libères, bien nez, bien intruictz, conversans en compaignies honnestes, ont par nature un instinct et aiguillon, qui toujours les poulse à faictz vertueux et retire de vice, lequel ilz nommoient honneur ».

27. Cligès, Les romans de Chrétien de Troyes, vol. 2, éd. Alexandre Micha, coll. « Les Classiques Français du Moyen Âge », Paris, H. Champion, 1982, v. 3123.

28. Pour les vers 382-87 de la Deuxième fille qui sont une rechristianisation des vers de l' « Ode à une aimée » de Sapho où le Paradis chrétien a remplacé le séjour des dieux antiques pour donner la mesure du bonheur d'être assis près de son ami, voir notre article «L'expression lyrique de la passion dans La comédie des quatre femmes (1542) de Marguerite de Navarre », Renaissance, Humanisme, Réforme, no 46, 1998, p. 40. 
136 / Renaissance and Reformation / Renaissance et Réforme

29. Anne Larsen a déjà relevé les endroits dans l'œuvre de Catherine Des Roches, que nous reproduisons ici, où la question de "vivre commodément », "sans amour » et «sans Serviteur » surgit ; voir Madeleine Des Roches, Catherine Des Roches, Les secondes auvres, éd. Anne R. Larson, Genève, Droz, 1998, p. 49. Ces affirmations surviennent tant dans ses œuvres de fiction, tel «Le Dialogue d'Iris et Pasithée ( Secondes æuuvres de 1583), que dans des ouvrages à caractère autoréférentiel, par exemple, dès «L'Épistre à sa Mere » dans Les CEuvres de 1579 et, à nouveau, en 1586, dans la lettre 42 des Missives. L'éditrice remarque aussi que «Les franches affirmations de l'érudite sont d'autant plus remarquables qu'à la Renaissance l'idée même d'une femme laïque, dont le désir serait de demeurer célibataire est inconcevable » (éd. cit., p. 49). Cette réflexion est approfondie dans « $\mathrm{La}$ réflexivité dans les dialogues de Catherine des Roches (1583) », in Dans les miroirs de l'écriture. La réflexivité chez les femmes écrivains d'Ancien Régime, éd. Jean-Philippe Beaulieu et Diane Desrosiers-Bonin, Montréal, Paragraphes, 1998, p. 61-71, où Anne Larsen fait un rapprochement entre les préoccupations de Catherine des Roches et celles d'Olympia Morata (1526/27-1555) dont les dialogues ont probablement inspiré la poitevine (p. 69-70), car bien que la célèbre humaniste de Ferrare, devenue protestante, se mariât, les remarques et attaques désobligeantes de son entourage, tant masculin que féminin, répétant que le temps consacré à se cultiver plutôt qu'à se parer l'empêcherait de trouver un mari, sont évoquées dans son Dialogue entre Lavinia della Rovere et Olympia Morata de 1550. Ce dialogue latin a été traduit en anglais dans Olympia Fulvia Morata, The Complete Writings of an Italian Heretic, éd. et trad. Holt N. Parker, Chicago, University of Chicago Press, 2003, p. 100-3.

Ceci soulève un autre problème, pris en compte par Évelyne Berrriot-Salvadore selon laquelle la Renaissance trouve le phénomène des filles prodiges fascinant, mais leur aspiration à devenir femmes savantes à jamais déconcertant. À ce sujet, elle donne l'exemple d'Étienne Pasquier « qui, tout en nourrissant une profonde admiration pour les poétesses poitevines, ne laisse pas de condamner, chez Catherine des Roches, son refus du mariage et cette volonté d'échapper à une destinée ordinaire, "ayant des livres et papiers qui lui feront perpétuelle compagnie” » (dans Marie de Gournay, Euvres complètes, éd. Jean-Claude Arnould, Évelyne Berriot, Claude Blum, Anna Lia Franchetti, Marie-Claire Thomine et Valerie Worth-Stylianou, vol. 1, Paris, H. Champion, 2002, p. 88). Cette question a été traitée de manière plus exhaustive par le même auteur dans « Les femmes dans les cercles intellectuels de la Renaissance : de la fille prodige à la précieuse », in Études corses, Études littéraires, Mélanges offerts au Doyen François Pitti-Ferrandi, Paris, Cerf, 1989, p. 210-37.

30. Pour éclairer la notion d'Anteros, souvent contradictoire et ambivalente, voir Robert V. Merrill, «Éros et Anteros », in Anteros. Actes du colloque de Madison (Wisconsin), mars 1994, éd. Ulrich Langer et Jan Miernowski, Orléans, Paradigme, 1994, p. 27-60, ainsi que les exemples commentés dans le même recueil de l'article de Jan Miernowski « Anteros face à face », p. 11-25, où ce dernier explique que dans un des poèmes de Pontus de Tyard, par exemple, Contr' amour signifie « Amour réciproque », alors que dans une de ses élégies, Anteros signifie «Contr' amour Dieu vengeur », mais parfois, ajoute-t-il, la métamorphose se produit à l'intérieur d'un même texte comme dans la série de dizains intitulée $L a$ distinction du vray amour de Marguerite de Navarre où l'on assiste à « Contr'amour conçu comme amor virtutis subjuguant le Cupidon de la passion charnelle » (p. 14-15).

31. V.-L. Saulnier, éd. cit., p. 85.

32. Bertrand de la Borderie, L'Amie de court (1542), éd. Danielle Trudeau, coll. « Textes de la Renaissance », 16, Paris, H. Champion. Nos citations sont tirées de cette édition. Notons 
que l'éditrice donne la graphie moderne pour amie, alors que Screech et Cottrell s'entendent sur la graphie de ce terme avec un y.

33. Le miroir des femmes, éd. Luce Guillerm et Jean-Pierre Guillerm, vol. 1 : Moralistes et polémistes au XVI siècle, Lille, Presses Universitaires de Lille, 1983, p. 193.

34. Voir Michael A. Screech, « An Interpretation of the Querelle des Amyes », Bibliothèque d'Humanisme et Renaissance, t. 21, 1959, p. 103-20 et tout spécialement p. 111, où Amye est qualifiée de «near-whore », ainsi que Robert D. Cottrell, « Le déplacement d'Éros par Anteros dans l'Amye de Court de La Borderie », in Langer et Miernowski, éd., op. cit., p. 117-36 et tout spécialement p. 134. Pour Cottrell, dans L'Amye de court, La Borderie oppose folle Amour à Vraye Amour ou honneste amytié, déplaçant progressivement Éros par son contraire Anteros, inspiré par Marot, qui oppose folle Amour à ferme Amour.

35. On conviendra que l'Amye de court se comporte de manière assez paradoxale. Après avoir invoqué «Saincte Diane » (La Borderie, p. 31, v. 521), la déesse rebelle à l'amour et protectrice des vierges et sa résolution de vivre comme elle « à jamais pudique et impolue » (p. 31, v. 526), elle raconte comment elle se soumet à l'épreuve de chasteté comme cette dame dont on raconte l'aventure dans Le livre du courtisan qui dormit nue pendant six mois dans les bras de son amant, pour prouver sa fermeté et sa constance.

36. D. Trudeau, éd. cit., p. xx pour la citation, ainsi que p. 63 et 65 pour l'anti-conformisme d'Amye comme «droit de façonner sa propre existence » et son anti-pétrarquisme. De même, comme l'a relevé Régine Reynolds-Cornell, il y a des échos de la Première fille et de la Seconde fille dans l'Heptaméron où on réentend chez Nomerfide «L'optimisme et l'espoir de bonheur de ceux dont les illusions n'ont pas encore été détruites »; voir Régine Reynolds, Les devisants de l'Heptaméron. Dix personnages en quête d'audience, Washington, DC, University Press of America, 1977, p. 130.

37. Gabrielle Suchon, Du célibat volontaire ou La vie sans engagement, 1700, éd. Sévérine Auffret, Paris, Indigo et Côté-femmes, 1994.

38. Les secondes auvres, éd. cit., p. 173, n. 33, où l'éditrice rapproche également ce passage du personnage de Corinna de Moderata Fonte dans Il merito delle donne (Venise, Imberti, 1600) où un même goût de la liberté et d'une vie sans serviteur traversera cette poésie.

39. Tilde Sankovitch, « The Dames des Roches. The Female Muse », in French Women Writers and the Book: Myths of Access and Desire, Syracuse, Syracuse University Press, 1988, p. 43-71, surtout p. 57 : « Chastity is for her less a matter of sexual than of intellectual and social status » et p. 58 : «For Catherine, chastity is a defense against male intrusion not only of the body but of the mind, and it is therefore indispensable for the access to the Book and book ».

40. Voir n. 23 supra pour l'étude de cette question chez Rabelais par Cioranescu.

41. Les secondes auvres, éd. cit., p. 98 et 99 , v. 1 et 9-14, pour le sonnet, et n. 32 ; voir aussi l'introduction, p. 12-13.

42. Moderata Fonte, op. cit., p. 49-50, pour le texte en italien et en anglais de ce sonnet dont nous traduisons les premières lignes. Voir aussi les commentaires de Virginia Cox (éd. cit., p. 50, n. 8) pour sa valeur de manifeste et les liens avec Marfisa.

43. À la question de savoir si la reine a une dette à l'endroit de l'Arioste, Pierre Jourda émet les plus grandes réserves afin de distinguer les parentés qui viendraient plutôt d'un fonds commun que d'un emprunt direct. Il écrit notamment à propos des rapports, évoqués par d'autres, entre la Nouvelle 20 de l'Heptaméron et un passage du Roland furieux sur Joconde qu'il y a identité du thème mais non dans le détail de chacune des phrases en laissant 
transparaître son incrédulité ainsi : «L'idée générale qui anime les deux passages est-elle si difficile à découvrir que Marguerite ait dû lire l'Arioste pour en tirer une nouvelle ?». Par contre, il est piquant qu'il compare la détermination de Marguerite à défendre François $\mathrm{I}^{\mathrm{er}}$, captif de Charles Quint, qui va jusqu'à inspecter les troupes, à celle des personnages de l'Arioste : «Elle joue les Amazones, ou, sans le savoir, les héroïnes de l'Arioste ou du Tasse » (Pierre Jourda, Marguerite d'Angoulême, duchesse d'Alençon, Reine de Navarre [1492-1549]. Étude biographique et littéraire, 2 vol., 1930, rééd. en fac-sim., Genève, Slatkine, 1978, v. 2, p. 705 et 1020).

44. Moderata Fonte, op. cit., p. 48-49. Outre cela, d'autres sont tentées de l'imiter en ne se mariant pas, sans oublier feue la riche tante de l'hôtesse Leonora qui a tellement aimé vivre seule et libre au point de faire du célibat le thème allégorique de son jardin.

45. Voici les parties saillantes de ce discours : « Je suis née libre, et c'est pour garder ma liberté que j'ai choisi la solitude des champs. [...] Jusqu'à présent, le ciel a décidé qu'il n'était pas de mon destin d'aimer ; il est inutile d'espérer que j'aime parce qu'on m'a choisie. [...] Si l'impatience et l'ardent désir de Chrysostome l'ont mené au tombeau, pourquoi en accuser ma réserve et l'honnêteté de ma conduite ? [...] Je possède, comme vous le savez, une fortune personnelle, et je ne convoite pas le bien d'autrui. J'ai le goût de la liberté et ne veux pas être asservie. Je n'aime ni ne hais personne. Je ne veux tromper celui-ci ni encourager celui-là ni me moquer de l'un ni m'amuser de l'autre. L'honnête conversation des bergères de ces villages et le soin de mes chèvres suffisent à m'occuper. Mes désirs ont ces montagnes pour limites ; et, s'ils vont au-delà, c'est pour contempler la beauté du ciel, montrant ainsi à mon âme le chemin de sa demeure première » (Miguel de Cervantes, L'ingénieux Hidalgo Don Quichotte de la Manche, trad. Aline Schulman, préf. de JeanClaude Chevalier, 2 vol., Paris, Seuil, 1997, vol. 1, ch. 14, p. 154-56). Que Don Quichotte, en sa qualité de chevalier, se porte à la défense de Marcelle pour appuyer son refus de répondre aux sollicitations de ses soupirants qui veulent la suivre sans égard pour les avertissements du morosophe est admirable. Mais le narrateur se demande ironiquement si ce sont ces menaces ou le fait que l'on n'avait pas fini d'enterrer Chrysostome qui fit que personne ne bougea.

46. Marie de Gournay, Le grief des Dames, Euvres complètes, éd. cit., t. 1, p. 1074-80. L'éditrice de ce texte, Évelyne Berriot-Salvadore, a noté que l'incipit du Grief des Dames reprend un passage de la Préface de 1595 de Mlle de Gournay aux Essais de Montaigne (ibid., t. 1, p. 283, n. 14). Pour la riche fortune et le développement de la revendication de la liberté spécifique de s'instruire et de se cultiver, v. Linda Timmermans, L'accès des femmes à la culture (1598-1715). Un débat d'idées de saint François de Sales à la Marquise de Lambert, Paris, H. Champion, 1993.

47. Élyane Dezon-Jones, Marie de Gournay. Fragments d'un discours féminin, Paris, Librairie José Corti, 1988, p. 13 ; voir aussi l'introduction sur les difficultés financières causées par les guerres civiles, mais aussi les procès liés à des questions d'héritage ainsi que les commentaires de l'auteur sur L'Apologie pour celle qui écrit (1641), où Marie de Gournay récuse les calomnies et autres atteintes à sa réputation mise à mal pour s'être écartée de la norme sociale. Voir aussi Marie le Jars de Gournay, Apology for the Woman Writing and Other Works, éd. et trad. Richard Hillman et Colette Quesnel, coll. « Other Voice in Early Modern Europe », Chicago, University of Chicago Press, 2002, p. 5-6 et p. 15-18, pour sa résistance aux projets de sa mère et sa détermination malgré un entourage critique à l'endroit de son désir d'être femme indépendante avec des ambitions intellectuelles. Dans un autre article, Richard Hillman se penche sur l'image de fille, puis de vieille fille imposée à Mlle de Gournay dès son vivant comme symptomatique des rapports inhérents à la nature humaine avec éros, car écarter cet état de fait « revient à reconnaître son emprise », dans 
«Les intellectuelles et l'amour: Marie de Gournay et Marguerite de Valois », Renaissance and Reformation / Renaissance et Réforme, t. 24, no 4, 2000, p. 125.

48. Gabrielle Suchon, Traité de la morale et de la politique. La Liberté, 1693, éd. Sévérine Auffret, Paris, Des femmes, 1988.

49. Il est instructif de comparer le Traité de Suchon avec les mises en garde répétées la même année de Jean Girard de Villethierry dans La vie des vierges ou Les devoirs et les obligations des vierges chrétiennes, devant les dangers qu'occasionne la volonté de liberté des filles. Celle-ci donnent, par la négative, une idée d'une certaine expansion qu'a prise le phénomène du célibat volontaire lequel, s'il ne peut être contré, sera contrôlé. Ainsi, Villethierry, l'esprit pétri par la contre-réforme rigoriste, oppose les distinctions de saint Basile sur les véritables Vierges qui ne doivent pas fuir le mariage pour circuler et vivre à leur guise, mais pour s'asseoir aux pieds de Jésus, leur « véritable Époux ». Ces célibataires, soustraites à l'autorité d'un mari, sont donc remises à cette autorité spirituelle comme si elles étaient entrées en religion. Ce livre, en confondant la plupart du temps virginité laïque et virginité sacerdotale, comme l'a observé son éditeur Constant Venesoen, fait de la maison un couvent, en voulant régler jusqu'à la nourriture et les sorties de ces malheureuses « invitées à connaître l'existence effacée des enterrées vivantes » (Jean Girard de Villethierry, La vie des vierges ou Les devoirs et les obligations des vierges chrétiennes, éd. Constant Venesoen, coll. «Textes de la Renaissance », 37, Paris, H. Champion, 2000, p. 27). Le refus d'accepter l'existence de la femme pour elle-même a réussi à dénaturer la possibilité de la virginité pieuse à la maison généreusement entrevue par Érasme (ibid., p. 61). L'éditeur a choisi comme texte de base la 5 édition revue et augmentée de 1714 de l'édition originale de 1693.

50. Le passage suivant est réminiscent de l'incipit du Grief des Dames de Mlle Gournay (bien que cette dernière ne soit jamais nommée comme tel), tout en manifestant un peu plus d'espoir dans les ressources du sexe. Gabrielle Suchon écrit : «Bien que les femmes et les filles soient en plusieurs manières privées du trésor de la liberté, tant par le malheur de leur condition qui les fait naître du Sexe le moins heureux, que par la sévérité des lois qui les tiennent toujours dans la contrainte, le caprice de la coutume a beau leur être contraire, il n'aura jamais le pouvoir d'abaisser leur esprit, d'enchaîner leur cœur, ni de rendre leur conscience criminelle» (éd. cit., p. 207).

51. Cette conception a l'originalité de comprendre les filles ainsi que les veuves qui choisissent cet état de femme seule, ce qui a l'avantage de situer les candidates à ce genre de vie selon leur inclination plutôt que la présence ou non d'un mari selon la tradition des trois âges de la femme.

52. Gabrielle Suchon, Du célibat volontaire, éd. cit., p. 134.

53. Simone de Beauvoir, Le deuxième sexe, 2 vol., Paris, Gallimard, 1949, vol. 1, p. 30 et 32.

54. L'imaginaire spéculatif surgit selon Claude-Gilbert Dubois, L'imaginaire de la Renaissance, coll. «Écriture », Paris, Presses Universitaires de France, 1985, p. 12 : « lorsque l'expérimentation d'idées procède par extrapolation du réel reconnu, par anticipation d'un réalisable, pour formuler des hypothèses ».

55. Le miroir des femmes, éd. cit., p. 193. 